\title{
TRIAD1 is negatively regulated by the MDM2 E3 ligase
}

\author{
SEUNGHEE BAE ${ }^{1}$, JIN HYUK JUNG ${ }^{1,2}$, IN-SOOK AN ${ }^{3}$, OK-YEOUN KIM ${ }^{3}$, MYOUNG JOO LEE $^{3}$, \\ JAE HO LEE ${ }^{4}$, IN-CHUL PARK ${ }^{5}$, SU-JAE LEE ${ }^{6}$ and SUNGKWAN AN ${ }^{1,3}$
}

\author{
${ }^{1}$ Molecular-Targeted Drug Research Center, Konkuk University, Gwangjin-gu, Seoul 143-701, Republic of Korea; \\ ${ }^{2}$ Section on Developmental and Stem Cell Biology, Department of Medicine, Joslin Diabetes Center, Harvard Medical School, \\ Boston, MA 02215, USA; ${ }^{3}$ Korea Institute for Skin and Clinical Sciences, Konkuk University, Gwangjin-gu, \\ Seoul 143-701; ${ }^{4}$ Laboratory of Molecular Oncology, Cheil General Hospital and Women's Healthcare Center, \\ Kwandong University, College of Medicine, Jung-gu, Seoul 100-380; ${ }^{5}$ Laboratory of Functional Genomics, \\ Korea Institute of Radiological and Medical Sciences, Nowon-gu, Seoul 139-706; \\ ${ }^{6}$ Department of Chemistry, Hanyang University, Seongdong-gu, Seoul 133-791, Republic of Korea
}

Received June 13, 2012; Accepted August 3, 2012

DOI: 10.3892/or.2012.2005

\begin{abstract}
Two RING fingers and DRIL1 (TRIAD1) is a proapoptotic protein that promotes p53 activation in several cancer cell lines, including MCF7, U2OS and A549 cells. In this study, we demonstrated that TRIAD1 is a novel ubiquitination target for proteasome-dependent degradation by murine double minute 2 (MDM2). TRIAD1 was found to interact with and be ubiquitinated by MDM2. RNA interference against MDM2 increased endogenous TRIAD1 protein stability. The functional study results suggested that TRIAD1 degradation by MDM2 suppresses TRIAD1-mediated cell growth. These data suggested a novel negative regulatory mechanism of TRIAD1 via MDM2 E3 ligase ubiquitination.
\end{abstract}

\section{Introduction}

Ubiquitin is a highly conserved small eukaryotic protein that is composed of 76 amino acids (1). The process of ubiquitination attaches ubiquitin to other proteins, and this is achieved by the enzymes E1, E2 and E3 ligase (1). Ubiquitination is an important post-translational modification that regulates protein qualities and quantities via proteasome-mediated degradation or changes in protein subcellular localization (2). Protein degradation through the ubiquitin-proteasome system is the major pathway for non-lysosomal proteolysis, and ubiquitin E3 ligases are responsible for tagging target proteins for degradation (2). There are hundreds of E3 ligases encoded in the human genome, and large studies regarding interplay between

Correspondence to: Dr Sungkwan An, Molecular-Targeted Drug Research Center, Konkuk University, 1 Hwayang-dong, Gwangjin-gu, Seoul 143-701, Republic of Korea

E-mail: ansfgrc@konkuk.ac.kr

Key words: p53, murine double minute 2, ubiquitination, two RING fingers and DRIL1 specific E3 ligases and their target proteins have been widely conducted. These investigations have demonstrated that dysregulation of the ubiquitin-proteasome system with regard to E3 ligases and their target proteins contributed to several diseases, such as cancer and neurodegenerative disorders (3). Therefore, understanding protein degradation and specific E3 ligases that control the process provides important insight into therapeutic approaches.

Two RING fingers and DRIL1 (TRIAD1) was originally found to play roles in differentiation and apoptosis (4-6). Previously, this protein was reported to be highly expressed during monocyte differentiation (4) and induced apoptosis in myeloid and cancer cell lines, including MCF7, A549, U2OS, H1299 and HCT116 (5,6). Moreover, the anticancer effect of TRIAD1 was mediated by $\mathrm{p} 53$ activation. Thus, it does not have any effect on p53-null cancer cells, while TRIAD1 ablation attenuated p53 transactivation (6). TRIAD1 was transcribed by HoxA10 transcription factor, which had an anticancer effect in acute myeloid leukemia (7). Notably, HoxA10 upregulated p53 expression and decreased breast cancer cell invasiveness (8). Collectively, this evidence suggested that additional investigations regarding TRIAD1 regulation would stimulate novel cancer therapeutic research, the mechanism, however, remains to be fully elucidated. In the present study, we demonstrated that murine double minute 2 (MDM2), a well-known E3 ligase for p53, also functioned as an ubiquitin E3 ligase for TRIAD1.

\section{Materials and methods}

Plasmids. Human TRIAD1 cDNA was cloned into pcDNA3.1$\mathrm{Myc} / \mathrm{His}$ (Invitrogen, Carlsbad, CA, USA), pEGFP-C1 (Clontech, Mountain View, CA, USA), and pGEX6p (Promega, Madison, WI, USA) vectors. Human MDM2 cDNA was cloned into the pCMV-Tag2-FLAG (Stratagene, La Jolla, CA, USA) vector. The RING domain-mutant MDM2 (MDM2-C438A) was created using the QuikChange site-directed mutagenesis kit (Stratagene), according to the manufacturer's instructions. The HA-ubiquitin (HA-Ub) plasmid pMT123 was kindly provided by Dr Dirk Bohmann (University of Rochester, 
Rochester, NY, USA). MDM2 small interfering RNA (siRNA) was purchased from Santa Cruz Biotechnology (Santa Cruz, CA, USA).

Antibodies and reagents. Antibodies against MDM2 (SMP14), TRIAD1 (F-23) and ubiquitin (P4D1) were from Santa Cruz Biotechnology. Anti- $\beta$-actin and anti-FLAG antibodies were from Sigma-Aldrich (St. Louis, MO, USA), anti-green fluorescent protein (GFP) antibody was from Cell Signaling Technology (Danvers, MA, USA) and GFP antibody for immunoprecipitation was from Anaspec (Fremont, CA, USA). CPT, etoposide, crystal violet, propidium iodine and MG132 were obtained from Sigma-Aldrich. Cycloheximide (CHX) was purchased from Biopure (Burlington, Ontario, Canada).

Cell culture and transfection. U2OS (KCLB no. 30096) cells were purchased from the Korean Cell Line Bank (Seoul, Korea). The $p 53^{-/} m d m 2^{-/}$MEF cell line was a kind gift from Dr Wei Gu (Columbia University, New York, NY, USA). $p 53^{-1} \mathrm{mdm} 2^{-1-}$ MEF cells were maintained in Dulbecco's minimum essential medium with $10 \%$ fetal bovine serum (FBS) and $1 \%$ penicillin/streptomycin (all from Invitrogen). U2OS cells were maintained in RPMI-1640 media (Invitrogen) with $10 \%$ FBS and $1 \%$ penicillin/streptomycin. Transient transfections were carried out using HilyMax (Dojindo Laboratories, Kumanoto, Japan).

Ubiquitination assays. $p 53^{-/} m d m 2^{-/-}$MEF cells were cotransfected with Myc-TRIAD1, HA-ubiquitin, FLAG-MDM2 or the FLAG-MDM2 RING mutant for $48 \mathrm{~h}$ and treated with $1 \mu \mathrm{M}$ MG132 for $12 \mathrm{~h}$. Cells were lysed with lysis buffer $(6 \mathrm{M}$ guanidine-HCl, $0.1 \mathrm{M} \mathrm{Na}_{2} \mathrm{HPO}_{4} / \mathrm{NaH}_{2} \mathrm{PO}_{4}$ and $10 \mathrm{mM}$ imidazole), sonicated and incubated with nickel-nitrilotriacetic acid (Ni-NTA, Qiagen) resin for $4 \mathrm{~h}$. Bound pellets were washed 6 times, using washing buffer $(25 \mathrm{mM}$ Tris- $\mathrm{HCl}, \mathrm{pH} 6.8$ and $20 \mathrm{mM}$ imidazole). The precipitates were then analyzed by immunoblotting, using an anti-HA (ubiquitin) antibody.

Immunoprecipitation and western blotting. For the immunoprecipitation assay, cell lysates in CHAPS buffer $(0.5 \%$ CHAPS; 10 mM Tris-HCl, $\mathrm{pH} 7.5 ; 1$ mM MgCl${ }_{2} ; 1$ mM EGTA; $5 \mathrm{mM} \beta$-mercaptoethanol; $10 \%$ glycerol) were incubated overnight with the indicated antibody with constant rotation at $4^{\circ} \mathrm{C}$. Following the addition of protein A/G PLUS-agarose beads (Santa Cruz Biotechnology), the cell lysates were incubated for an additional $2 \mathrm{~h}$ followed by extensive washing. For the immunoblotting assay, cells were lysed in RIPA buffer $(10 \mathrm{mM}$ Tris-HCl, $\mathrm{pH} 7.4 ; 150 \mathrm{mM} \mathrm{NaCl}_{2} ; 1 \%$ NP-40; $1 \%$ deoxycholate; and $0.1 \%$ SDS) containing a protease inhibitor cocktail (Roche Applied Sciences, Indianapolis, IN, USA). Proteins were electrophoresed and transferred onto a nitrocellulose membrane, which was incubated overnight with each indicated antibody at $4^{\circ} \mathrm{C}$. For protein detection, horseradish peroxidaseconjugated anti-mouse or anti-rabbit antibodies (Cell Signaling Technology) were used, followed by enhanced chemiluminescence (ECL; Pierce, Thermo Fisher Scientific, Rockford, IL, USA) and autoradiography.

Cell viability assays. The cell proliferation ratio was measured using the 3-(4,5-dimethylthiazol-2yl)-5-(3-carboxymethoxy-
phenyl)-2-(4-sulfophenyl)-2H-tetrazolium inner salt (MTS) assay kit (Promega), according to the manufacturer's instructions. Briefly, cells were transfected with GFP, GFP-TRIAD1 or FLAG-MDM2, plated onto 96-well culture plates at a density of 1,000 cells/well and incubated for $48 \mathrm{~h}$. The MTS/PMS solution was added to each well and incubated at $37^{\circ} \mathrm{C}$ for $1-2 \mathrm{~h}$. The absorbance was measured at $490 \mathrm{~nm}$ using a microplate reader. The cell proliferation ratio relative to that of the control cells was graphically represented.

Colony formation assays. Cells seeded on 6-well plates (at $4 \times 10^{4}$ cells/well) were transfected with GFP, GFP-TRIAD1, or FLAG-MDM2 plasmids. Subsequent to a 2-week incubation, colonies were fixed and stained with $0.1 \%$ crystal violet.

Statistical analysis. Statistical analysis was performed using the two-tailed Student's t-test. $\mathrm{p}<0.05$ was considered to indicate a statistically significant difference.

\section{Results}

TRIAD1 ubiquitination and degradation is induced by MDM2 E3 ligase. TRIAD1-mediated apoptosis has been previously reported to be induced by p53 activation (6). Occasionally, proteins responsible for $\mathrm{p} 53$ activation, such as RUNX3, ribosome protein L11 and p300, might be targets for MDM2 E3 ligase (9-11). Therefore, TRIAD1 was examined as a potential novel ubiquitination target for MDM2. To test this hypothesis, the protein level of TRIAD1 upon MDM2 overexpression was initially examined. Notably, MDM2 greatly decreased TRIAD1 protein levels in a MDM2 concentration-dependent manner (Fig. 1A). MDM2 lacking RING ligase activity was also found not to have induced TRIAD1 degradation (Fig. 1A), indicating that this process was MDM2 E3 ligase activity-dependent. These results led to our examining whether MDM2-mediated TRIAD1 degradation was handled by the proteasomal degradation system. The MDM2-mediated TRIAD1 decrease was found to have markedly recovered when proteasomal function was inhibited with MG132 (Fig. 1B), indicating that MDM2 induces the proteasomal degradation of TRIAD1. Furthermore, immunoprecipitation assays demonstrated an interaction between TRIAD1 and MDM2 in cells (Fig. 1C), suggesting that TRIAD1 is a direct ubiquitination target for MDM2 E3 ligase. We also assessed whether TRIAD1 is ubiquitinated by MDM2. As expected, TRIAD1 was determined to have been ubiquitinated by MDM2 via its RING ligase activity (Fig. 1D). These results indicated that TRIAD1 was a novel target for MDM2 E3 ligase and was subsequently degraded via ubiquitin-mediated proteolysis.

TRIAD1 protein stability is regulated by MDM2 E3 ligase. TRIAD1 cellular stability has not been evaluated previously. The above results of MDM2-induced TRIAD1 degradation led to our examining whether the regulation of exogenous and endogenous TRIAD1 protein stability was a result of MDM2 E3 ligase activity. The exogenous TRIAD1 stability in an MDM2 overexpression system was examined and results showed that the half-life of the TRIAD1 protein was decreased in an MDM2 E3 ligase activity-dependent manner (Fig. 2A). Conversely, the stability of endogenous TRIAD1 was increased following 
A

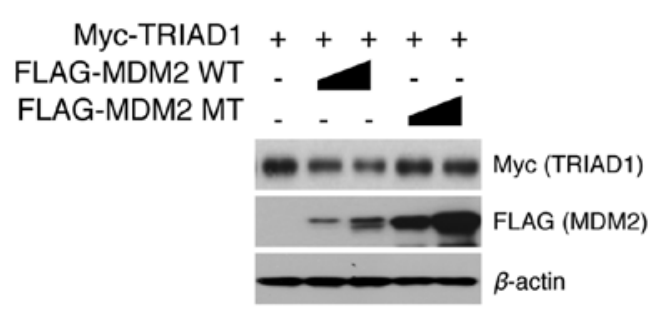

B

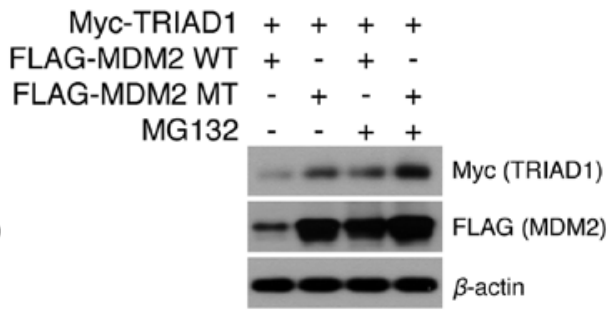

C

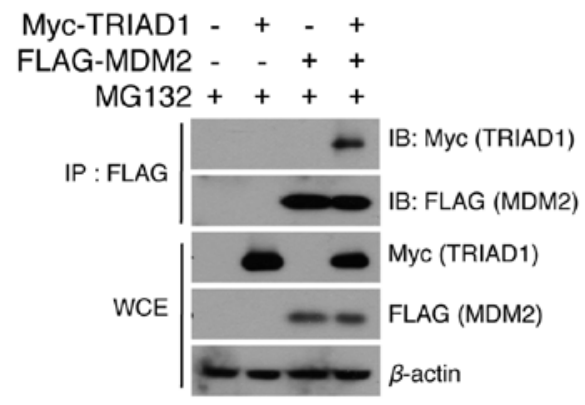

D

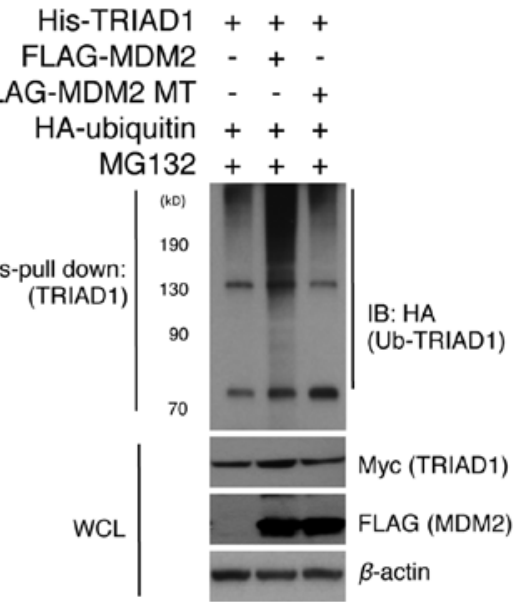

Figure 1. TRIAD1 is ubiquitinated by MDM2 E3 ligase. (A) MDM2 decreases the level of ectopically expressed TRIAD1. $p 53^{-/} \mathrm{mdm} 2^{-/-} \mathrm{MEF}$ cells were transfected with Myc-TRIAD1, WT FLAG-MDM2 or RING-domain mutant MDM2 (MDM2 MT). Immunoblotting was performed with the indicated antibodies $24 \mathrm{~h}$ after transfection. (B) MDM2-mediated degradation of TRIAD1 was abrogated in the presence of proteasome inhibitor. $\mathrm{p5} 3^{-/} \mathrm{mdm} / \mathrm{m}^{-/} \mathrm{MEF}$ cells were cotransfected with Myc-TRIAD1 and either WT or MT FLAG-MDM2 plasmids. Twenty-four hours after transfection, the cells were treated with $1 \mu \mathrm{M}$ MG132 for $12 \mathrm{~h}$. Cell lysates were subjected to immunoblotting with anti-Myc antibody. (C) TRIAD1 interacts with MDM2. $p 53^{-/} \mathrm{mdm} 2^{-/}$MEF cells were cotransfected with FLAG-MDM2 and Myc-TRIAD1 expression plasmids. Twenty-four hours after transfection, the cells were treated with MG132 for $4 \mathrm{~h}$ and anti-FLAG immunoprecipitates were subjected to immunoblotting using anti-Myc antibody. Whole cell lysates (WCL) were analyzed by immunoblotting with the indicated antibodies as a control. (D) TRIAD1 is an ubiquitination target for MDM2. $p 53^{--} \mathrm{mdm} 2^{--}$MEF cells were cotransfected with Myc/His-TRIAD1 and HA-ubiquitin in combination, with either WT or MT FLAG-MDM2 plasmid. Twenty-four hours after transfection, the cells were treated with $10 \mu \mathrm{M}$ MG132 and then incubated for $4 \mathrm{~h}$. The cells were lysed and pulled down using Ni-NTA agarose resin. TRIAD1 ubiquitination was examined by immunoblotting with anti-Myc antibody. Ectopic TRIAD1 and FLAG-MDM2 expression levels were analyzed by immunoblotting with the indicated antibodies.

\section{A}
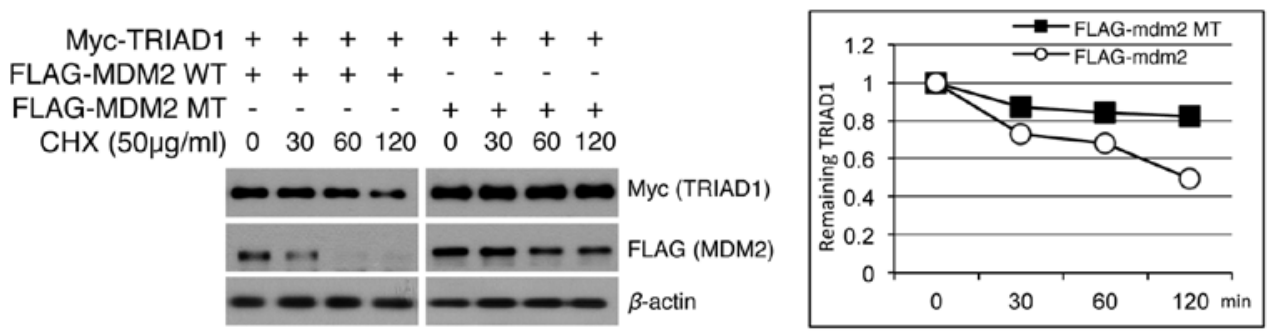

B

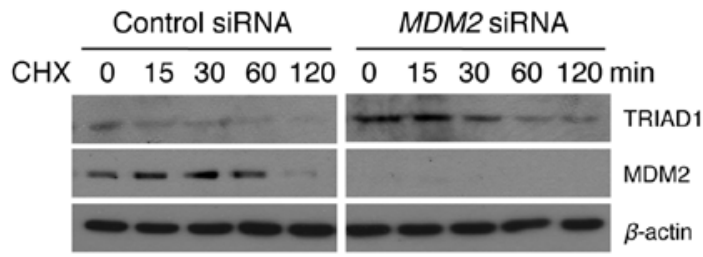

Figure 2. TRIAD1 stability is regulated by MDM2 E3 ligase. (A) TRIAD1 stability is decreased by ectopic MDM2 expression. $p 53^{-/} \mathrm{mdm} 2^{-/-}$MEF cells were cotransfected with Myc-TRIAD1 and WT or MT FLAG-MDM2. Twenty-four hours after transfection, the cells were treated with cycloheximide (CHX, $150 \mu \mathrm{g} / \mathrm{ml}$ ) for the indicated times. Levels of TRIAD1 were measured by immunoblotting, and residual levels of TRIAD1 were quantitated by densitometry. The half-life of TRIAD1 was calculated based on the decay of normalized (to $\beta$-actin) p53 levels to $50 \%$ of their original level. (B) Stability of endogenous TRIAD1 increases upon MDM2 knockdown. U2OS cells were transfected with control or MDM2 siRNA. Twenty-four hours after transfection, the cells were treated with cycloheximide (CHX, $150 \mu \mathrm{g} / \mathrm{ml})$ for the indicated times. Levels of TRIAD1 were measured by immunoblotting. 
A

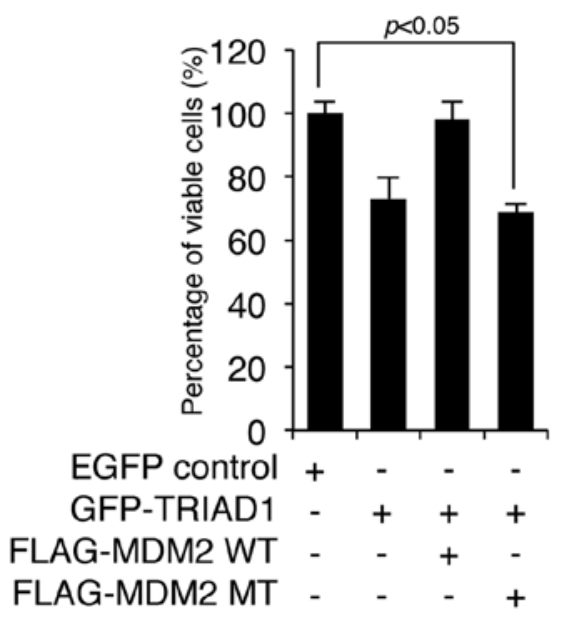

B

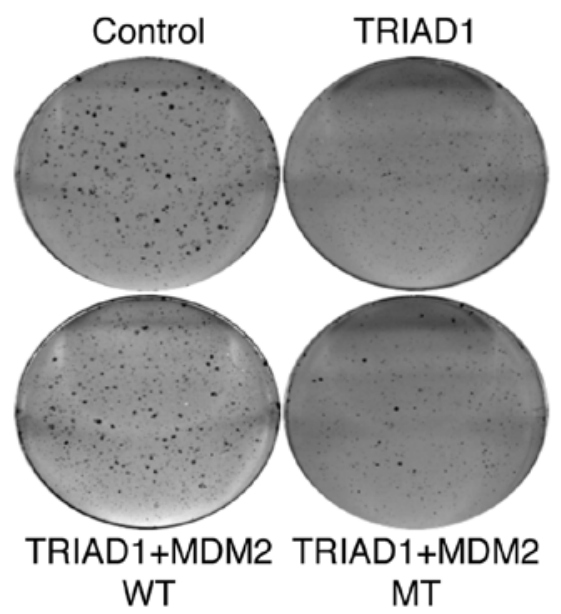

Figure 3. TRIAD1-mediated cellular growth inhibition is attenuated by MDM2. (A) U2OS cells were cotransfected with either EGFP control plasmid or a plasmid encoding GFP-TRIAD1, along with WT or MT FLAG-MDM2. After $72 \mathrm{~h}$ of incubation, the cell viability was measured using the MTS assay. Error bars show the SEM ( $\mathrm{n}=6$ ). The results were statistically significant compared with the control cells for each condition (p<0.05). (B) TRIAD1-mediated clonogenic growth inhibition is abrogated by MDM2. U2OS cells were transfected with plasmids for Myc-TRIAD1 and WT or MT FLAG-MDM2, incubated in media containing G418 (500 $\mu \mathrm{g} / \mathrm{ml})$ for 2 weeks and stained with crystal violet.

MDM2 knockdown (Fig. 2B). Collectively, these results suggest that TRIAD1 was regulated by MDM2 E3 ligase in vitro.

MDM2 attenuates TRIAD1-mediated cell growth inhibition. Since E3 ligase usually negatively regulates the activity of its substrates (12), we measured the cell growth following TRIAD1 transfection in the presence or absence of wild-type (WT) or mutant (MT) MDM2 to test whether MDM2 negatively regulated TRIAD1-mediated cell growth inhibition. As shown in Fig. 3A, TRIAD1-mediated cell growth inhibition was completely blocked by WT, although not MT MDM2, which lacks RING ligase activity. Furthermore, TRIAD1-mediated clonogenic growth inhibition was reduced by MDM2 (Fig. 3B). These results indicate that MDM2 negatively regulated TRIAD1 function via its RING ligase activity.

\section{Discussion}

Results of the present study have demonstrated the novel regulatory mechanism of the TRIAD1 protein by MDM2 E3 ligase. TRIAD1 was found to have interacted with MDM2 in vivo. Although the precise region responsible for the interaction was not identified in this study, the results suggest that TRIAD1 is potentially an ubiquitination substrate for MDM2. TRIAD1 was ubiquitinated and targeted for proteasomal degradation in an MDM2-dependent manner. Conversely, MDM2 siRNA increased the stability of endogenous and exogenous TRIAD1. Cell proliferation and colony-forming assays demonstrated that the antiproliferative function of TRIAD1 was inhibited by MDM2. Overall, the above data indicate that TRIAD1 function is regulated by an ubiquitin-dependent proteasomal pathway through MDM2 E3 ligase.

Although TRIAD1 has been previously reported to have induced apoptosis via p53 activation (6), the molecular interplay between TRIAD1 and MDM2 has been determined as non-p53 status-dependent, since, in the present study, TRIAD1 was ubiquitinated and degraded by MDM2 in p53-null MEF cells. However, the antiproliferative function of TRIAD1 depends on the p53 status, since TRIAD1 overexpression in p53-null MEF or p53-null HCT116 cells did not induce apoptosis [data not shown; (6)]. Therefore, although MDM2-induced TRIAD1 degradation was independent of p53, the physiological role of TRIAD1-MDM2 is mediated by p53. Furthermore, although MDM2 directly inhibits p53 stability and activity (13), MDM2 is also believed to negatively regulate p53 activation by inhibiting TRIAD1 for the following reasons: i) TRIAD1 induces apoptosis via p53 activation (6); ii) the ablation of TRIAD1 attenuates p53 transactivation (6) and iii) TRIAD1 degradation by MDM2 is independent of $\mathrm{p} 53$, while negative MDM2-induced TRIAD1 cellular function regulation is p53-dependent. Thus, a balance between TRIAD1 and the MDM2 expression may be the primary factor involved in p53 regulation.

ARF is a tumor suppressor frequently deleted in several tumors (14-16). Both ARF and TRIAD1 activate p53 (17). ARF induces MDM2 degradation (18), whereas TRIAD1-mediated MDM2 regulation is not clearly understood. TRIAD1 and ARF may synergistically mediate p53 activation, although no such mechanism has been elucidated. However, if ARF were not required for TRIAD1-mediated p53 activation, TRIAD1 would be a novel tumor therapeutic target given that ARF is deleted in several types of cancer (14-16).

Based on these results, a novel regulatory mechanism of TRIAD1-MDM2 is proposed, whereby TRIAD1 is regulated by MDM2 via ubiquitin-mediated proteolysis. The finding that TRIAD1 induces p53 activation emphasizes the importance of the development of p53-mediated tumor therapeutic targets.

\section{Acknowledgements}

The authors would like to thank Dr B.A. van der Reijden (Radboud University Nijmegen Medical Centre, The Netherlands), for providing the GFP-TRIAD1 plasmids. The 
authors are also grateful to the members of the research group, for their support and suggestions regarding this study. The present study was financed by the 2011 Konkuk University research support program.

\section{References}

1. Hershko A and Ciechanover A: The ubiquitin system. Annu Rev Biochem 67: 425-479, 1998.

2. Mani A and Gelmann EP: The ubiquitin-proteasome pathway and its role in cancer. J Clin Oncol 23: 4776-4789, 2005.

3. Ardley HC and Robinson PA: E3 ubiquitin ligases. Essays Biochem 41: 15-30, 2005.

4. van der Reijden BA, Erpelinck-Verschueren CA, Lowenberg B and Jansen JH: TRIADs: a new class of proteins with a novel cysteine-rich signature. Protein Sci 8: 1557-1561, 1999.

5. Marteijn JA, van Emst L, Erpelinck-Verschueren CA, et al: The E3 ubiquitin-protein ligase Triad1 inhibits clonogenic growth of primary myeloid progenitor cells. Blood 106: 4114-4123, 2005.

6. Jung JH, Lee SM, Bae S, et al: Triad 1 induces apoptosis by $\mathrm{p} 53$ activation. FEBS lett 584: 1565-1570, 2010.

7. Wang H, Bei L, Shah CA, Horvath E and Eklund EA: HoxA10 influences protein ubiquitination by activating transcription of ARIH2, the gene encoding Triad1.J Biol Chem 286: 16832-16845, 2011.

8. Chu MC, Selam FB and Taylor HS: HOXA10 regulates p53 expression and matrigel invasion in human breast cancer cells. Cancer Biol Ther 3: 568-572, 2004.

9. Chi XZ, Kim J, Lee YH, et al: Runt-related transcription factor RUNX3 is a target of MDM2-mediated ubiquitination. Cancer Res 69: 8111-8119, 2009.
10. Dai MS, Shi D, Jin Y, et al: Regulation of the MDM2-p53 pathway by ribosomal protein L11 involves a post-ubiquitination mechanism. J Biol Chem 281: 24304-24313, 2006.

11. Jin Y, Zeng SX, Lee H and Lu H: MDM2 mediates p300/CREBbinding protein-associated factor ubiquitination and degradation. J Biol Chem 279: 20035-20043, 2004.

12. Leng RP, Lin Y, Ma W, et al: Pirh2, a p53-induced ubiquitinprotein ligase, promotes p53 degradation. Cell 112: 779-791, 2003.

13. Momand J, Zambetti GP, Olson DC, George D and Levine AJ: The mdm-2 oncogene product forms a complex with the p53 protein and inhibits p53-mediated transactivation. Cell 69: 1237-1245, 1992.

14. Faderl S, Kantarjian HM, Estey E, et al: The prognostic significance of $\mathrm{p} 16(\mathrm{INK} 4 \mathrm{a}) / \mathrm{p} 14(\mathrm{ARF})$ locus deletion and MDM-2 protein expression in adult acute myelogenous leukemia. Cancer 89: 1976-1982, 2000.

15. Fulci G, Labuhn M, Maier D, et al: p53 gene mutation and ink4a-arf deletion appear to be two mutually exclusive events in human glioblastoma. Oncogene 19: 3816-3822, 2000.

16. Pasmant E, Laurendeau I, Heron D, Vidaud M, Vidaud D and Bieche I: Characterization of a germ-line deletion, including the entire INK4/ARF locus, in a melanoma-neural system tumor family: identification of ANRIL, an antisense noncoding RNA whose expression coclusters with ARF. Cancer Res 67: 3963-3969, 2007.

17. Midgley CA, Desterro JM, Saville MK, et al: An N-terminal p14ARF peptide blocks Mdm2-dependent ubiquitination in vitro and can activate p53 in vivo. Oncogene 19: 2312-2323, 2000.

18. Zhang Y, Xiong Y and Yarbrough WG: ARF promotes MDM2 degradation and stabilizes p53: ARF-INK4a locus deletion impairs both the $\mathrm{Rb}$ and $\mathrm{p} 53$ tumor suppression pathways. Cell 92: 725-734, 1998 